\title{
ON THE GENERALIZED HURWITZ-LERCH ZETA FUNCTION AND GENERALIZED LAMBERT TRANSFORM
}

\author{
VIRENDRA KUMAR
}

\begin{abstract}
Raina and Srivastava [20] introduced a generalized Lambert transform. Goyal and Laddha [8] have introduced generalizations of the Riemann zeta function and generalized Lambert transform. In the present paper, we introduce generalizations of the Hurwitz-Lerch zeta function and Lambert transform in a diverse direction. We derive generating functions involving generalized Hurwitz-Lerch zeta function. Connections between the generalized Lambert transform and generalized Hurwitz-Lerch zeta function are established. An inversion formula for the generalized Lambert transform is obtained. Some examples and special cases to illustrate our results are also mentioned.
\end{abstract}

Mathematics subject classification (2010): 11M06, 11M35, 33C10.

Keywords and phrases: Riemann zeta function, Hurwitz-Lerch zeta function, special functions, generating functions, Lambert transform, Mellin inversion theorem.

\section{REFERENCES}

[1] B. R. Bhonsle, A relation between Laplace and Hankel transforms, Proc. Glasgow Math. Assoc. 5, 3 (1962), 114-115.

[2] B. R. BHONSLE, A relation between Laplace and Hankel transforms, Math. Japon. 10 (1965), 85-89.

[3] A. Erdélyi, W. Magnus, F. Oberhettinger and F. G. Tricomi, Higher Transcendental Functions, Vol I, McGraw-Hill Book Company, New York, Toronto and London, 1953.

[4] A. Erdélyi, W. Magnus, F. Oberhettinger and F. G. Tricomi, Higher Transcendental Functions, Vol II, McGraw-Hill Book Company, New York, Toronto and London, 1953.

[5] K. C. Gupta And S. M. AgRawal, Unified theorems involving $H$-function transform and Meijer Bessel function transform, Proc. Indian Acad. Sci. (Math. Sci.) 96, 2 (1987), 125-130.

[6] S. P. Goyal And S. K. VAsishta, Certain relations between generalized Kontorovitch-Lebdev transform and $H$-function transform, Ranchi Univ. Math. Jour. 6 (1975), 95-102.

[7] S. P. GOYAL AND R. M. JAIN, Certain results for two-dimensional Laplace transform with applications, Proc. Nat. Acad. Sci. India, 59 (A), (III) (1989), 407-414.

[8] S. P. Goyal AND R. K. LAdDHA, On the generalized Riemann zeta functions and the generalized Lambert transform, Ganita Sandesh 11, 2 (1997), 99-108.

[9] P. Humbert And R. P. Agarwal, Sur la fonction de Mittag-Leffler et quelques unes de ses generalizations, Bull. Sci. Math. 77, 2 (1953), 180-185.

[10] V. KumaR, A general class of functions and $N$-fractional calculus, J. Rajasthan Acad. Phys. Sci. 11, 3 (2012), 223-230.

[11] V. KUMAR, $N$-fractional calculus of general class of functions and Fox's $H$-function, Proc. Natl. Acad. Sci., sect. A, Phys. Sci. 83, 3 (2013), 271-277.

[12] V. Kumar, The Euler transform of $V$-function, Afr. Mat. 29, (1-2) (2018), 23-27.

[13] V. Kumar, Theorems connecting Mellin and Hankel transforms, J. Classical Anal. 15, 2 (2019), $123-130$.

[14] V. KumaR, Theorems connecting Fourier sine transform and Hankel transform, SeMA Journal 77, 2 (2020), 219-225.

[15] V. KumAR, Theorems connecting Stieltjes transform and Hankel transform, São Paulo J. Math. Sci. doi:10.1007/s40863-020-00182-4. 
[16] V. Kumar, On a General Theorem Connecting Laplace Transform and Generalized Weyl fractional Integral Operator Involving Fox's H-Function and a General Class of Functions, J. Frac. Calc. and Appl. 11, 2 (2020), 270-280.

[17] V. Kumar, On a Generalized Fractional Fourier Transform, Palestine J. Maths. 9, 2 (2020), 903-907.

[18] G. M. Mittag-LeffleR, Sur la nouvelle function $E_{\alpha}(x)$, C. R. Acad. Sci. Paris 137 (1903), 554 558.

[19] T. R. PrabhaKAR, A singular integral equation with a generalized Mittag-Leffler function in the kernel, Yokohama Math. J. 19 (1971), 7-15.

[20] R. K. Raina AND H. M. SRIVASTAVA, Certain results associated with the generalized Riemann zeta functions, Rev. Tecn. Fac. Ingr. Univ. Zulia 31, 3 (1995), 301-304.

[21] H. M. SRIVASTAVA, A relation between Meijer and Hankel transforms, Math. Japon. 11 (1966), 11 13.

[22] H. M. SRIVAstaVA, On a relation between Laplace and Hankel transforms, Matematiche (Catania) 21 (1966), 199-202.

[23] H. M. SRIVAstaVa, Some remarks on a generalization of the Stieltjes transform, Publ. Math. Debrecen 23 (1976), 119-122.

[24] H. M. SRIVAstava, K. C. Gupta AND S. P. Goyal, The H-function of one and two Variables with Applications, South Asian Publishers, New Delhi, 1982.

[25] H. M. SRIVAstaVA AND V. K. TuAn, A new convolution theorem for the Stieltjes transform and its application to a class of singular integral equations, Arch. Math. (Basel) 64, 2 (1995), 144-149.

[26] H. M. SRIVASTAVA AND O. YÜREKLI, A theorem on a Stieltjes-type integral transform and its applications to obtain infinite integrals of elementary and special functions, Complex Vari., Theory Appl. 28, 2 (1995), 159-168.

[27] T. O. SALIM, Some properties relating to the generalized Mittag-Leffler function, Advances in Applied Mathematical Analysis 4 (2009), 21-30.

[28] E. C. TitchmaRCH, Introduction to the Theory of Fourier Integrals, Clarendon Press, Oxford, 1948.

[29] A. Wiman, Über den Fundamentalsatz in der Teorie der Funktionen $E_{\alpha}(x)$, Acta. Math. 29 (1905), 191-201.

[30] E. M. Wright, The asymptotic expansion of the generalized Bessel function, Proc. London Math. Soc. 38, 2 (1935), 257-270.

[31] D. V. WIDDER, An inversion of the Lambert transform, Math. Mag. 23 (1950), 177-182.

[32] S. Yakubovich And M. Martins, On the iterated Stieltjes transform and its convolution with applications to singular integral equations, Trans. Spec. Func. 25, 5 (2013), doi:10.1080/10652469.2013.868457. 